\title{
Functional MRI with reduced susceptibility artifact: high-resolution mapping of episodic memory encoding
}

\author{
Peter Fransson, ${ }^{1,2}$ Klaus-Dietmar Merboldt,' Martin Ingvar, ${ }^{3}$ Karl Magnus Petersson ${ }^{3}$ and \\ Jens Frahm ${ }^{\text {I,CA }}$
}

\begin{abstract}
'Biomedizinische NMR Forschungs GmbH am Max-Planck-Institut für biophysikalische Chemie, 37070 Göttingen, Germany; ${ }^{2}$ MR Research Center and ${ }^{3}$ Cognitive Neurophysiology R2-0I, Department of Clinical Neuroscience, Karolinska Institutet/Karolinska Hospital, Stockholm, Sweden

${ }^{\mathrm{CA}}$ Corresponding Author
\end{abstract}

Received I4 February 200I; accepted I March 200I

\begin{abstract}
Visual episodic memory encoding was investigated using echoplanar magnetic resonance imaging at $2.0 \times 2.0 \mathrm{~mm}^{2}$ resolution and $1.0 \mathrm{~mm}$ section thickness, which allows for functional mapping of hippocampal, parahippocampal, and ventral occipital regions with reduced magnetic susceptibility artifact. The memory task was based on 54 image pairs each consisting of a complex visual scene and the face of one of six different photographers. A second group of subjects viewed the same set of images without memory instruction as well as a reversing checkerboard. Apart from visual activation in occipi-
\end{abstract}

tal cortical areas, episodic memory encoding revealed consistent activation in the parahippocampal gyrus but not in the hippocampus proper. This finding was most prominently evidenced in sagittal maps covering the right hippocampal formation. Mean activated volumes were $432 \pm 293 \mu \mathrm{l}$ and $259 \pm 179 \mu \mathrm{l}$ for intentional memory encoding and non-instructed viewing, respectively. In contrast, the checkerboard paradigm elicited pure visual activation without parahippocampal involvement. NeuroReport 12:1415-1420 (C) 200I Lippincott Williams \& Wilkins.

Key words: Hippocampus; Human brain; Magnetic resonance imaging; Memory; Neuroimaging; Parahippocampus

\section{INTRODUCTION}

The exact role of the hippocampus and the medial temporal lobe in memory formation is still an open issue [1,2]. This not only refers to the fact that hippocampal involvement has been ascribed to a large variety of different memory functions [3] but also relates to questions regarding a functional segregation and specialization along its anterior-posterior axis [4,5]. With respect to neuroimaging, complications emerge from technical limitations such as low spatial resolution, e.g. using PET, or the presence of marked susceptibility-induced signal losses and geometric distortions in long echo time gradient-echo MRI, as commonly employed for functional mapping of deoxyhemoglobin-mediated brain responses.

The purpose of this work was to exploit the potential of a recently developed version of echo-planar imaging (EPI) that allows for a reduction of susceptibility artifacts while providing access to high spatial resolution [6]. The approach rninimizes the intravoxel phase dispersion and corresponding signal loss in $\mathrm{T}^{*}$-weighted EPI of the hippocampus by acquiring thin sections of only $1 \mathrm{~mm}$ thickness at an in-plane resolution of $2.0 \times 2.0 \mathrm{~mm}^{2}$ that is by reducing the voxel size during data acquisition to $4 \mu \mathrm{l}$.
This voxel size is at least one order of magnitude smaller than most previous attempts to functional mapping of memory [7-10]. It is therefore expected to provide reliable responses in most peri-hippocampal areas except perhaps the anterior-inferior medial temporal lobe.

The visual memory task studied here bears two major elements. The paradigm contains the concept of novelty [11-13] in comparing presentations of visual scenes unknown to the subject with seeing a familiar gray screen with a central fixation cross. Moreover, the task involves aspects of associative learning or relational declarative memory [14-17] as the subjects were instructed to encode image pairs relating a complex scene with the face of its photographer. Because of the methodologic aspects of this study, both of these elements were incorporated into the experimental paradigm in order to maximize differential blood oxygenation-level dependent (BOLD) MRI responses between conditions. To investigate whether intentional memory encoding and attentive viewing activate different brain regions, a control group viewed the same set of image pairs without the memory instruction. In addition, the control group viewed a reversing checkerboard as a second low-level cognitive reference condition. 


\section{MATERIALS AND METHODS}

A total of 16 healthy volunteers participated in this project. Excluding the data of two subjects for technical reasons, eight subjects (25-35 years, mean $29 \pm 3$ years) were studied for the memory task and six subjects (19-32 years, mean $24 \pm 4$ years) for the visual control paradigms. Informed written consent was obtained from all volunteers prior to the examinations. All studies were conducted at $2.0 \mathrm{~T}$ using a conventional MRI system (Siemens Vision, Erlangen, Germany) equipped with $25 \mathrm{mT} \mathrm{m}^{-1}$ gradients. Images were acquired with use of the standard head coil.

MRI: Dynamic BOLD MRI was based on a single-shot blipped gradient-echo EPI sequence (TE $=54 \mathrm{~ms}$, symmetrical coverage of $\mathrm{k}$ space, $1470 \mathrm{~Hz}$ bandwidth per pixel) using a $5.34 \mathrm{~ms}$ slice-selective RF pulse to achieve highquality profiles for interleaved acquisitions of 16 contiguous sections at $1 \mathrm{~mm}$ thickness. T2*-weighted images were obtained with flip angle of $70^{\circ}$ at $\mathrm{TR}=2000 \mathrm{~ms}$ temporal resolution to minimize the influence of $\mathrm{T} 1$ saturation and inflow effects. In all cases the in-plane resolution was $2.0 \times 2.0 \mathrm{~mm}^{2}$ using a data matrix of $112 \times 128$ together with a $7 / 8$ rectangular field of view of $224 \times 256 \mathrm{~mm}^{2}$. For further details see [6]. Here, the hippocampal region was covered by a $16 \mathrm{~mm}$ volume along its long axis in both an oblique transverse orientation and, following the right hippocampal formation, in an oblique sagittal orientation.

For data analysis and presentation of the results in form of activation maps the 16 dynamic data sets of $1 \mathrm{~mm}$ thickness were grouped into four contiguous sections of $4 \mathrm{~mm}$ effective thickness by adding the raw images from neighboring acquisitions. The resulting images are shown in Fig. 1 for both the transverse and sagittal orientation and all four sections. Because these $\mathrm{T} 2 *$-weighted images rather than the anatomical images commonly selected for background purposes determine the true reliability of the activation maps, it seems mandatory to demonstrate their general quality and regional access. This particularly applies to brain areas that are critically affected by magnetic field inhomogeneities.

Paradigms: Presentation of visual stimuli was accomplished by means of a projection set up (Schäfter and Kirchhoff, Hamburg, Germany) covering $30 \times 40^{\circ}$ of the subject's visual field. The study involved two groups of subjects and three different paradigms. The first group of subjects performed an intentional memory encoding task. The visual material comprised 54 colored image pairs showing a complex photographed scene together with the face of one of six different photographers as an insert. The control condition was to view a gray screen of homogenous isoluminescent gray light with a central red cross as a fixation point. Room lights were switched on during all examinations providing background illumination.

Before the MRI examination, all subjects were shown examples of the image pairs (not used in the experiments) and familiarized with the gray light stimulus. They were instructed to look attentatively to the stimulus material and to memorize the presented image pairs. They were informed that their memory performance were to be evaluated immediately after MRI scanning. It was assessed in a multiple forced choice recognition paradigm, that is

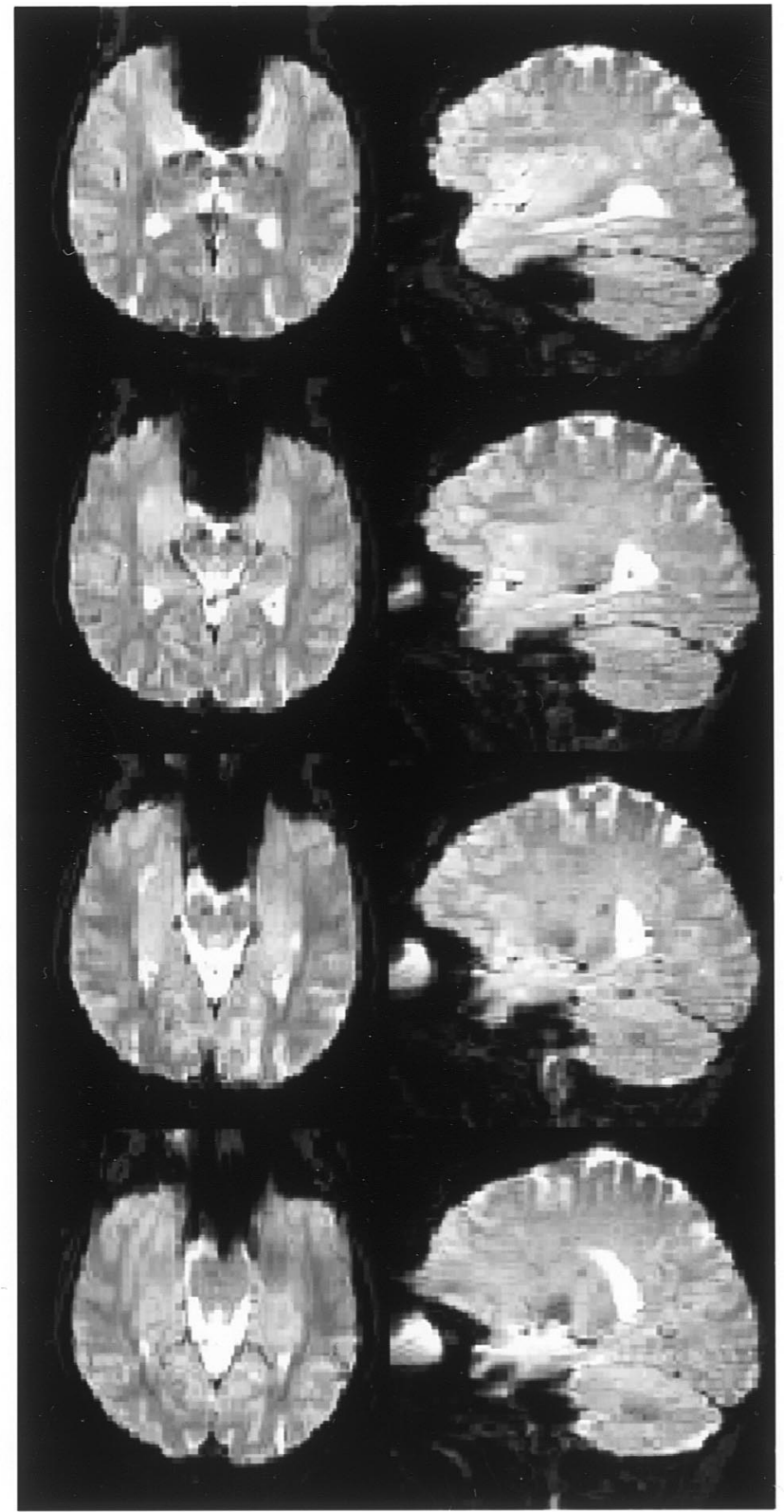

Fig. I. Transverse-to-coronal (left) and sagittal (right) images of consecutive sections of $4 \mathrm{~mm}$ thickness covering contiguous $16 \mathrm{~mm}$ volumes of the base of the brain and the right temporal lobe, respectively. All images were originally acquired as 16 contiguous I mm EPI sections (TR/ $\mathrm{TE}=2000 / 54 \mathrm{~ms}$, flip angle $70^{\circ}$, in plane resolution $2.0 \times 2.0 \mathrm{~mm}^{2}$ ) to reduce the degree of intravoxel dephasing and concomitant susceptibility artifacts.

the subjects were confronted with the same set of 54 photographed images (randomized order) and a template that contained the faces of all six photographers. The subjects had to use a computer mouse to pick the photographer they remembered to have been shown together with each particular scene during MRI.

A second group of subjects viewed the same 54 image pairs without a memory instruction. They also viewed a reversing checkerboard stimulus (frequency $5 \mathrm{~Hz}$ ) which 
consisted of a circular, radial arrangement of 16 wedges formed by seven to eight black and white segments at equal radial distance. In both cases, the control condition was a gray screen with a central red fixation cross.

The temporal structure of all paradigms was based on a block design and followed the physiologic response characteristics previously investigated in this laboratory $[18,19]$. Individual image pairs were presented for a period of $4 \mathrm{~s}$ (i.e. corresponding to the duration of two image acquisitions) using six cycles of $12 \mathrm{~s}$ memory encoding (three image pairs) and $18 \mathrm{~s}$ of gray screen. To establish baseline conditions the six cycles were preceded by a $20 \mathrm{~s}$ period of the control condition.

Data analysis: The analysis of dynamic MRI data sets involved neither spatial or temporal smoothing nor any other kind of preprocessing as, for example, baseline or drift corrections. Functional responses to task-related changes in brain activity were identified by cross-correlation, which compares the MRI signal intensity time courses of each pixel with a box-car reference function that mimics the temporal structure of the paradigm shifted by $4 \mathrm{~s}$ with respect to stimulus onset to account for hemodynamic latencies. An initial region of interest analysis of signal intensity time courses from different regions in the medial temporal lobe gave no indication of responses with other, e.g. longer-lasting, temporal characteristics.

Quantitative maps of correlation coefficients were obtained by a fully automated and user-independent statistical analysis based on in-house software following the ideas outlined in [20]. The approach estimates the individual noise distribution underlying the histogram of each correlation coefficient map and then rescales the correlation coefficients as percentile ranks of the noise distribution. Highly significant centers of activation are identified by accepting all pixels $>99.99 \%$ percentile rank of the individual noise distribution of correlation coefficients. This upper threshold corresponds to an error probability of $p<0.0001$ or $1-2$ false positive pixels per map. Improved delineation of coherently activated areas is achieved by iteratively adding directly neighboring pixels provided their correlation coefficients exceed a lower threshold corresponding to the $95 \%$ percentile rank of the noise distribution. For a quantitative assessment of activated volumes the final maps were independently read by an experienced neuroradiologist and a psychiatrist specialized in studies of the hippocampal system.

\section{RESULTS}

The mean percentage of correctly remembered image pairs in the intentional memory encoding task was $60 \% \pm 19 \%$ (averaged across subjects) contrasting a rate of $16.7 \%$ for pure chance, that is six possible choices for each scene. In the case of attentive viewing, subjects were interviewed with regard to their overall recollection of the presented material but no quantitative evaluation was performed.

Figure 2 shows four consecutive transverse to coronal activation maps obtained for the episodic memory encoding task for two subjects in the left. and right column, respectively. They reveal activation in the ventral stream of the occipital cortex as well as in a region in the right posterior part of the parahippocampal gyrus in the lowest

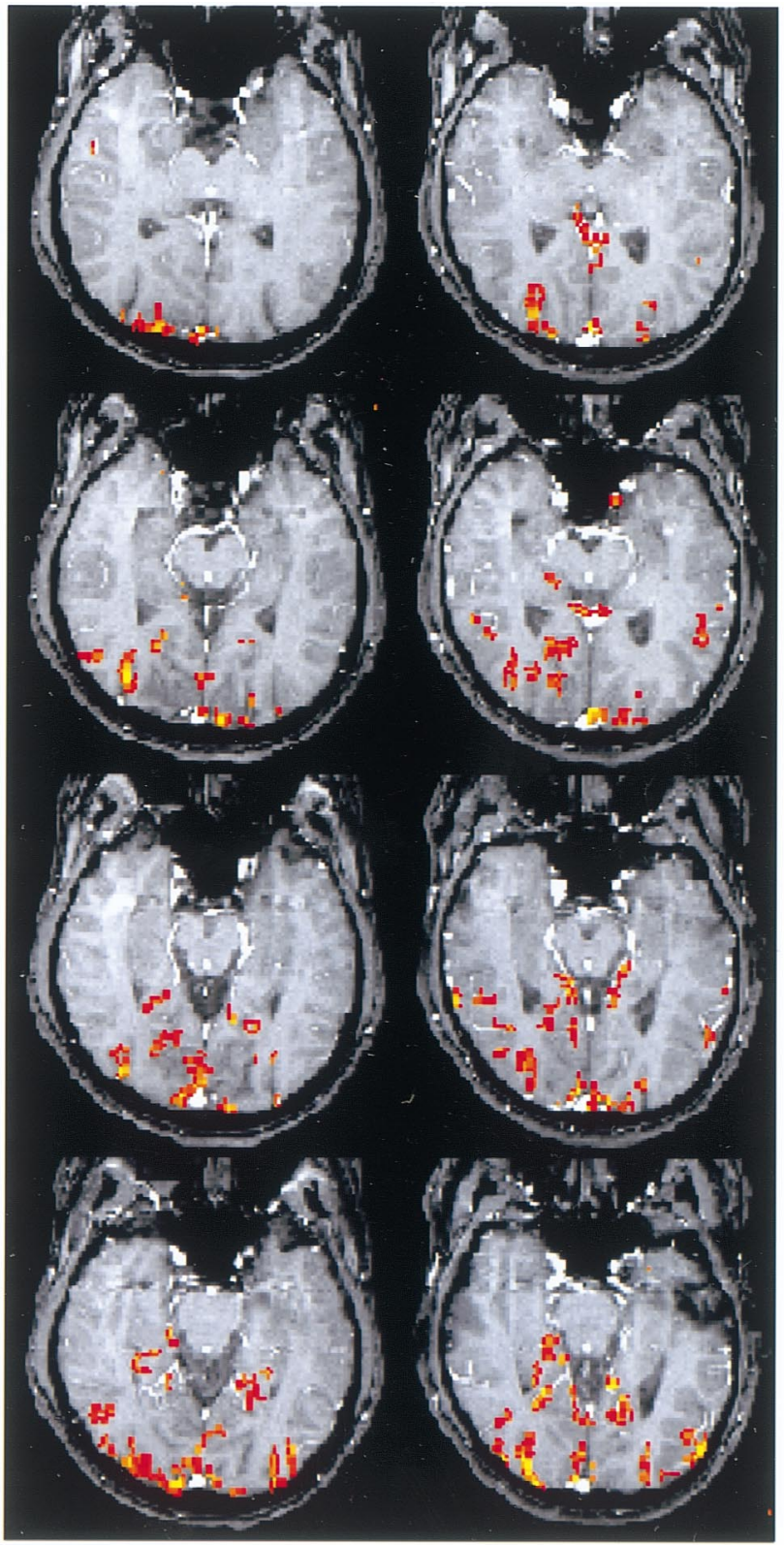

Fig. 2. Functional activation in response to episodic memory encoding in two subjects (left and right column, respectively) using a transverse-tocoronal orientation. The data in the left column originates from the same subject as shown in the left column of Fig. I. Apart from visual areas, activation occurs in a region posterior to the right hippocampus in the lowest sections (bottom images).

sections (right hemispheres correspond to the left side of the images). These were general findings in all subjects. Further details emerge from activation maps covering the right hippocampal formation in a sagittal orientation as shown in Fig. 3 for two different subjects. They clearly demonstrate that, again apart from visual areas in occipital cortex, activation in relation to episodic memory encoding is confined to regions in the parahippocampal gyrus. It is noteworthy that the high spatial resolution of these maps unambiguously separates the parahippocampal gyrus and 


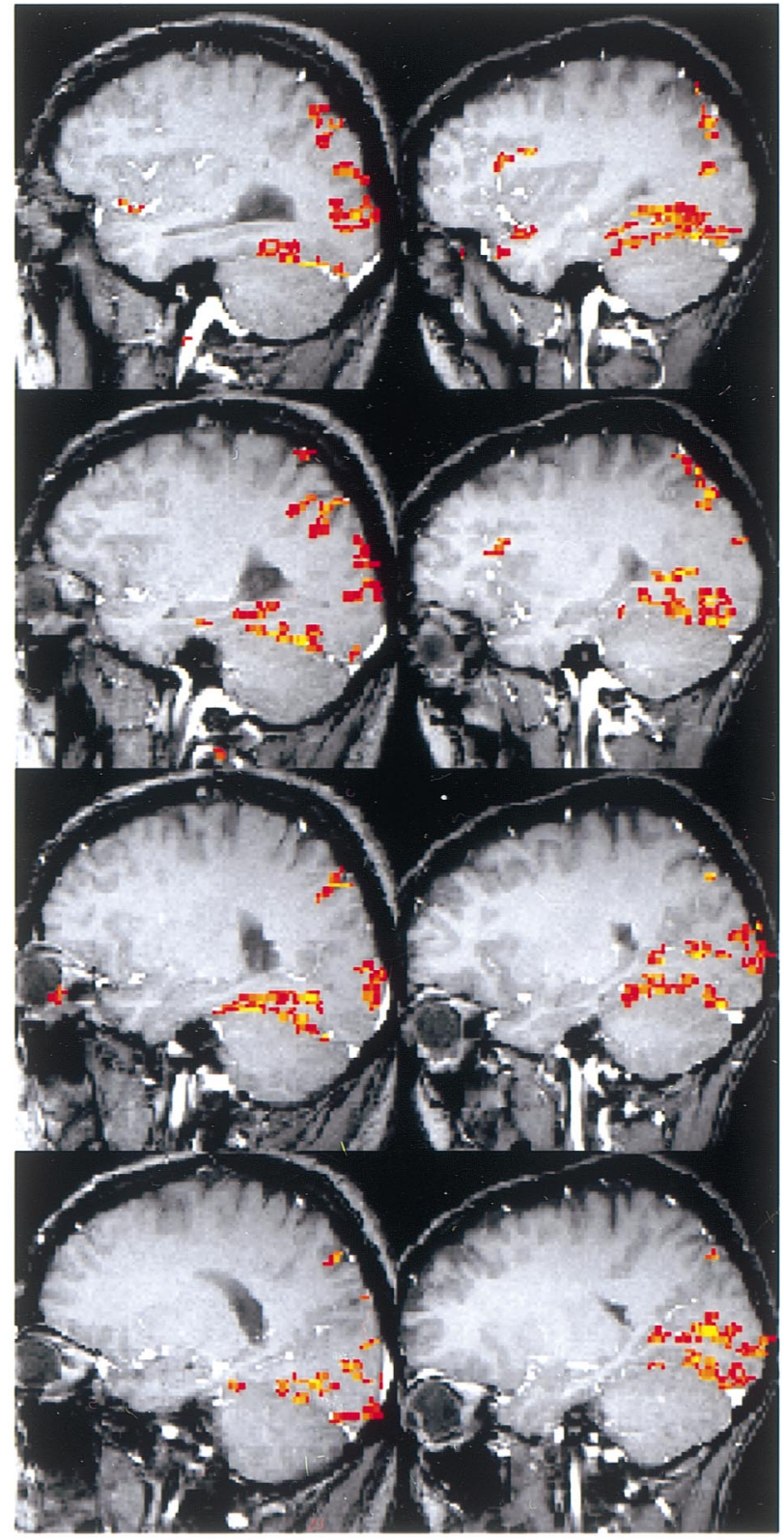

Fig. 3. Functional activation in response to episodic memory encoding in two different subjects from those shown in Fig. 2 (left and right column, respectively) using a sagittal orientation covering the right medial temporal lobe. The data in the left column originates from the same subject as shown in the right column of Fig. I. In agreement with Fig. 2 and apart from visual areas, activation occurs in the right parahippocampal gyrus but not in the hippocampus proper. the hippocampus proper, indicating an absence of activation in the hippocampus proper. A quantitative analysis of the spatial extent of activation is summarized in Table 1. The values represent the number of activated pixels averaged across subjects and sections and demonstrate a statistically significant $(p<0.05)$ lateralization to the right parahippocampal gyrus for intentional memory encoding (see also [21]).

Viewing of the same image pairs as used for intentional memory encoding as well as visual stimulation by a checkerboard paradigm were studied in a second group of subjects. A key finding is the observation that incidentally seeing complex scenes leads to activation of the same region posterior to the right hippocampus in transverse to coronal activation maps (not shown) as observed for intentionally memorizing the same material. This is confirmed and even more clearly demonstrated in Fig. 4, which presents the corresponding sagittal activation maps covering the right medial temporal lobe. Whereas activation for a reversing checkerboard is confined to occipital areas (right column), attentive viewing of complex visual material causes parahippocampal activation (left column) similar to that seen in Fig. 3 for intentional memory encoding. Again, Table 1 provides a more detailed analysis of respective activation volumes. Because non-instructed viewing does not lead to a lateralization but causes an equivalent involvement of the right and left parahippocampus, the right parahippocampal gyrus shows a statistically non-significant trend for reduced activation compared to intentional memory encoding with corresponding volumes of $259 \pm 179 \mu \mathrm{l}$ vs $432 \pm 293 \mu \mathrm{l}$, respectively.

\section{DISCUSSION}

The present methodology offers BOLD MRI studies with substantially reduced susceptibility artifact in most areas of the medial temporal lobe, that is in sections covering the hippocampus and parahippocampal gyrus. Possible exceptions may still be regions in the anterior-inferior portion of the most medial temporal lobe. Although the chosen technique is at the expense of volume coverage and for a $2 \mathrm{~s}$ temporal resolution offers only 16 sections at $1 \mathrm{~mm}$ thickness, its reduced susceptibility artifact and excellent resolution allows an unambiguous distinction between activations within different regions of the hippocampal and parahippocampal domain.

The paradigm for visual memory encoding was based on our previous experiments $[16,22]$ as well as on literature findings [11-14]. It was selected for this first application of the thin section fMRI method [6] to maximize the differential work load on this memory encoding network rather

Table I. Parahippocampal activation for intentional memory encoding and non-instructed viewing of visual material.

\begin{tabular}{lll}
\hline Region & Intentional encoding & Non-instructed viewing \\
\hline Right parahippocampal gyrus (transverse sections) & $29.3 \pm 20.9(n=6)$ & $15.7 \pm 13.9(n=6)$ \\
Left parahippocampal gyrus (transverse sections) & $2.2 \pm 3.5(n=6)$ & $12.3 \pm 14.6(n=6)$ \\
Right parahippocampal gyrus (transverse and sagittal sections) & $27.0 \pm 18.3(n=8)$ & $16.2 \pm 11.2(n=12)$ \\
\hline
\end{tabular}

Values are mean \pm s.d. number of pixels averaged across subjects and sections. The size of a pixel corresponds to a volume of $16 \mu \mathrm{l}$. 


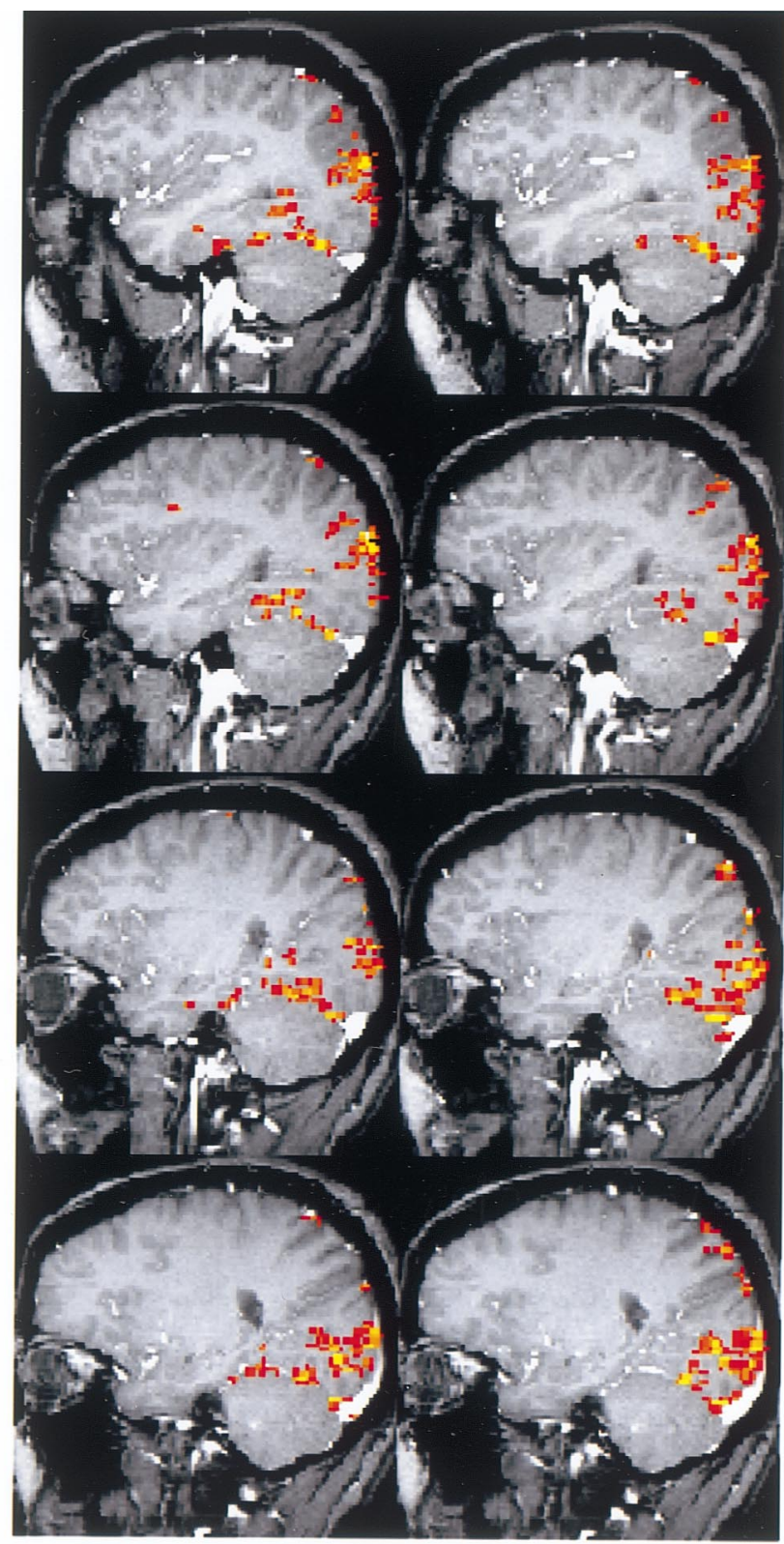

Fig. 4. Functional activation in response to (left) non-instructed viewing of the same complex images as employed for the intentional encoding and (right) reversing checkerboard stimulation in a single subject using a sagittal orientation covering the right medial temporal lobe. Apart from visual areas, incidental memory encoding of complex scenes elicits activation in the right parahippocampal gyrus which vanishes for checkerboard stimulation.

than to elucidate specific aspects of episodic memory encoding such as novelty and associative learning. Hence, the decision to use a familiar gray screen as a control condition, which in the context of memory encoding may be considered to be a familiar low-level baseline condition, should be viewed as an attempt to enhance the contrast between a non-encoding vs an encoding situation. Taking these provisions into account it seems that the present encoding task does not differentially activate the right or left hippocampus but involves the right parahippocampal gyrus. Of course, it may be argued that the absence of hippocampal activation may be a consequence of insufficient sensitivity or functional contrast or even reflect an altered hemodynamic response function so that activations escaped the applied correlation analysis with a reference function similar to that used for other brain areas. However, a search for BOLD MRI responses with different time constants as well as an attempt to lower the risk of false negatives by setting the threshold to $p<0.0005$ (uncorrected) failed as far as the present data are concerned. In addition, we also made technical attempts to exclude direct vascular, that is flow-related, signal enhancements as an erroneous source of activation.

Taking into account the high spatial resolution achieved in this study, our finding of parahippocampal activation for episodic memory encoding is in agreement with preceding low-resolution MRI studies of visual associative tasks and novel picture encoding that lead to activations in the medial temporal lobe. For example, the present findings clearly extend reports of activation in the posterior hippocampal formation and parahippocampal gyrus at an effective spatial resolution of $6.25 \times 6.25 \times 7.0 \mathrm{~mm}^{3}$ [12], in posterior portions of the parahippocampal region as well as in the hippocampal formation at $4.0 / 6.1 \times 3.0 \times 5.0 \mathrm{~mm}^{3}$ effective resolution [23], and in a posterior medial-temporal region that were focused in the parahippocampal cortex [24] by specifically identifying the right posterior parahippocampal gyrus as a key area for episodic memory encoding of visual information. Whether the use of low spatial resolution together with post-acquisition low-pass spatial filtering and intersubject averaging artificially merges activations in the hippocampal and parahippocampal areas even for the same data as presented here, is the subject of an alternative analysis currently in progress. Without doubt such procedures preclude a detailed regional analysis when the final maps approach a spatial accuracy of 8$10 \mathrm{~mm}$ in all three dimensions [22].

Viewing complex images without a memory instruction activates similar posterior regions in the right, parahippocampal gyrus as found for explicit memory encoding, whereas checkerboard stimuli with only basic visual elements confined activations to visual occipital areas. This finding is consistent with a recent PET study of encoding and retrieval of verbal material that demonstrated involvement of the parahippocampal gyrus for both intentional and incidental memory tasks [25]. The most likely explanation for our observation is the hypothesis that attentive visual processing of complex scenes automatically recruits memory functions as an integral part of this cognitive enterprise (see also [26]). This is also supported by the observation that all subjects were able to recall pertinent scenes when interrogated after the experiments.

\section{CONCLUSION}

The ability to acquire reliable functional maps of a major portion of the hippocampal formation at high spatial resolution presents a most relevant contribution to the ongoing endeavour of understanding the mechanisms of human memory. Functional neuroimaging with a voxel size of $2 \times 2 \times 1 \mathrm{~mm}^{3}$ as opposed to typically $3 \times 3 \times 5 \mathrm{~mm}^{3}$ provides the resolution required for an examination of 
hippocampal and parahippocampal regions as spatially distinct structures processing a cognitive task. In particular, sagitta1 activation maps convincingly demonstrate that the parahippocampal gyrus is engaged in intentional episodic memory encoding of visual information as well as in noninstructed viewing of complex scenes which includes elements of incidental episodic memory encoding. With the assumption that the hemodynamic properties in the hippocampus are similar to those in other brain areas, we did not observe differential encoding-related activity in the hippocampus proper but in the parahippocampal region. Although at lower spatial resolution, this failure to detect significant hippocampal activation in encoding and retrieval tasks has also been reported by others [1].

\section{REFERENCES}

1. Fletcher PC, Frith CD and Rugg MD. TINS 20, 213-218 (1997).

2. Tulving E and Markowitsch HJ. Curr Op Neurobiol 7, 209-216 (1997).

3. Cohen NJ, Ryan J, Hunt C et al. Hippocampus 9, 83-98 (1999).

4. Lepage M, Habib R and Tulving E. Hippocampus 8, 313-322 (1998).

5. Schacter DL, Curran T, Reiman EM et al. Hippocampus 9, 575-581 (1999).

6. Merboldt KD, Finsterbusch J and Frahm J. J Magn Reson 145, 184-191 (2000).

7. Brewer JB, Zhao Z, Desmond JE et al. Science 281, 1185-1187 (1998).

8. Fernández G, Weyerts H, Schrader-Bölsche M et al. J Neurosci 18,
1841-1847 (1998).

9. Wagner AD, Schacter DL, Rotte M et al. Science 281, 1188-1191 (1998).

10. Dolan RJ and Fletcher PC. Hippocampus 9, 25-34, (1999).

11. Tulving E, Markowitsch. RJ, Kapur S et al. Neuroreport 5, 2525-2528 (1994).

12. Stern CE, Corkin S, Gonzáles G et al. Proc Natl Acad Sci 93, 8660-8665 (1996),

13. Dolan RJ and Fletcher PC. Nature 388, 582-585 (1997).

14. Henke K, Buck A, Weber B et al. Hippocampus 7, 249-256 (1997).

15. Eichenbaum H. Аnnu Rev Psychol 48, 547-572 (1997).

16. Montaldi D, Mayes AR, Barnes A et al. Hum Brain Mapp 6, 85-104 (1998).

17. Petersson KM, Reis A, Castro-Caldas A et al. Neurolmage 10, 45-54 (1999).

18. Fransson P, Krüger G, Merboldt KD et al. Magn Reson Med 39, 912-919 (1998).

19. Fransson P, Krüger G, Merboldt KD et al. Neuroreport 9, 2001-2005 (1998).

20. Kleinschmidt A, Requardt M, Merboldt KD et al. Int J Imag Syst Technol 69, 238-244 (1995).

21. Kelley WM, Miezin FM, McDermott KB et al. Neuron 20, 927-936 (1998).

22. Petersson KM, Nichols TE, Poline JB et al. Phil Trans R Soc Lond B 354, 1261-1281 (1999).

23. Rombouts SA, Machielsen WC, Witter MP et al. Hippocampus 7, 594-601 (1997).

24. Gabrieli JDE, Brewer JB, Desmond JE et al. Science 276, 264-266 (1997).

25. Rugg MD, Fletcher PC, Frith CD et al. Neuroreport 8, 1283-1287 (1997).

26. Morris RG and Frey U. Phil Trans Royal Soc Lond B 352, 1489-1503 (1997).

Acknowledgements: P.F., K.M.P. and M.I. were supported by grants from the Swedish Medical Research Concil (8246), the Swedish Bank Tercentennial Foundation, the Knut and Alice Wallenberg Foundation and the Stockholm County Council. We thank Drs Ingrid Agartz and Dan Greltz for an independent assessment of hippocampal and parahippocampal volumes. 